\title{
Development of a 2D Image Reconstruction and Viewing System for Histological Images from Multiple Tissue Blocks: Towards High-Resolution Whole-Organ 3D Histological Images
}

\author{
Noriaki Hashimoto ${ }^{a}$ Pinky A. Bautista ${ }^{b}$ Hideaki Haneishi $^{a}$ Matija Snuderl $^{d}$ \\ Yukako Yagi ${ }^{b, c}$ \\ ${ }^{a}$ Center for Frontier Medical Engineering, Chiba University, Chiba, Japan; ${ }^{b}$ Department of Pathology, Massachusetts \\ General Hospital, and 'Department of Pathology, Harvard Medical School, Boston, Mass., and d New York University \\ Langone Medical Center, New York, N.Y., USA
}

\section{Key Words}

2D image reconstruction $\cdot$ Histology image $\cdot$ Whole organ power of a standard computer used in our laboratories is of interest. We also present the initial implementation of our 2D image viewer system in this paper. $2016 \mathrm{~S}$. Karger AG, Basel

\begin{abstract}
High-resolution 3D histology image reconstruction of the whole brain organ starts from reconstructing the high-resolution 2D histology images of a brain slice. In this paper, we introduced a method to automatically align the histology images of thin tissue sections cut from the multiple paraffinembedded tissue blocks of a brain slice. For this method, we employed template matching and incorporated an optimization technique to further improve the accuracy of the $2 \mathrm{D}$ reconstructed image. In the template matching, we used the gross image of the brain slice as a reference to the reconstructed 2D histology image of the slice, while in the optimization procedure, we utilized the Jaccard index as the metric of the reconstruction accuracy. The results of our experiment on the initial 3 different whole-brain tissue slices showed that while the method works, it is also constrained by tissue deformations introduced during the tissue processing and slicing. The size of the reconstructed high-resolution 2D histology image of a brain slice is huge, and designing an image viewer that makes particularly efficient use of the computing
\end{abstract}

\section{Introduction}

Studies on multimodal image registrations of medical images have been conducted to investigate how the different imaging modalities represent the same tissue region as image signals and how these signals can be related to each other [1-3]. Multimodal registration of medical images, when successful, can lead to the discovery of diagnostic metrics, which are helpful in histopathology diagnosis.

In pathology, stained slides of sectioned tissues are prepared from pieces of tissue specimens excised from tissue organs. Pathologists then make their diagnosis by observing these stained slides under a microscope. The locations of the excised specimens with respect to the $3 \mathrm{D}$ volume of the whole organ and the locations of the sectioned tissues in the excised specimen are vital information for the accurate registration of histology images with

\section{KARGER}

(c) 2016 S. Karger AG, Basel

$1015-2008 / 16 / 0833-0127 \$ 39.50 / 0$

E-Mail karger@karger.com

www.karger.com/pat
Yukako Yagi, $\mathrm{PhD}$

Pathology Imaging and Communication Technology Center

Massachusetts General Hospital, 101 Merrimac Street, Suite 820

Boston, MA 02114 (USA)

E-Mail YYAGI@mgh.harvard.edu 
respect to images obtained using other modalities, such as computer tomography (CT) or magnetic resonance (MR). However, pathology reports do not necessarily contain this type of information. 3D histology image reconstruction of the whole tissue organ could lend information on the locations and orientations of the excised specimens in other modal images.

Whole-slide imaging (WSI) technology, which enabled us to capture the high-resolution images of stained tissue sections, has not only encouraged the development of various automated histopathology image analysis systems for diagnostic supports [4-12], the technology has also spurred interest in 3D histology image reconstruction of tissue organs [13-17]. Reconstruction of the 3D volumetric image of a tissue organ from series of histology images is an emerging application area in digital pathology. Initial works in this area have concluded that a $3 \mathrm{D}$ histology image volume representation of a tissue organ or of an excised tissue specimen has the potential for discovering novel morphological and growth patterns of human disease. The 3D reconstruction of a tissue organ from its histology images faces a unique challenge. Series of histology images, in which some might exhibit tissue tearing due to inconsistency in tissue sectioning or folding due to imperfect mounting of the sectioned tissues onto the glass slides, have to be precisely aligned. Tissue tearing or folding is inevitable when manual tissue processing is adapted. Fortunately, using the recent technology innovation on automated tissue sectioning systems, which can provide us with hundreds of high-quality serial tissue sections, reconstruction of the $3 \mathrm{D}$ image of a tissue specimen is easier and more accurate [18]. The reconstructed 3D histology image of a tissue organ, or of an excised tissue specimen, can be viewed using the existing $3 \mathrm{D}$ image viewer software [19]. The 3D viewer will enable us to explore the surfaces of the sectioned tissues at various orientations and at various magnifications up to the original scanned magnification.

A research group in Germany reconstructed the 3D histology image volume of an entire tissue organ using whole mount histological sections [20]. Using whole mount tissue sections has some issues to wit. Large glass slides used for mounting the whole histological sections are not routinely used in histology laboratories. Besides, special equipment and special technician skills are required to process whole mount tissue sections. Moreover, the image quality of thick tissue sections, such as the whole mount tissue sections, is subpar in comparison to the quality of thin tissue sections [21]. That is, the 3D image volume created from whole mount tissue sections of- fers limited cellular details of the tissue sections. Acquiring the high-resolution histology image of whole mount tissue sections presents another challenge since the current WSI systems available in the market were generally designed to image histology sections which were mounted on standard-size glass slides. Clearly, in implementing the high-resolution 3D histology image reconstruction of a whole tissue organ it is advantageous to utilize thin tissue sections that are mounted on standard-size glass slides. An added benefit of using thin tissue sections is that the present workflow in pathology laboratories will not be disrupted when preparing the histology slides for our 3D histology projects.

Initial works on high-resolution 3D histology image reconstruction considered a single formalin-fixed paraffin-embedded (FFPE) tissue block [13-17]. Series of thin tissue sections were cut from the FFPE tissue blocks, mounted on standard-size glass slides and then stained. Although the reconstructed $3 \mathrm{D}$ volume of the single FFPE tissue block offers only partial 3D visualization of the entire organ, it still has great potential for discovering novel metrics for diagnosis, which is not possible with $2 \mathrm{D}$ histology images. In fact, it was shown $[13,15]$ that novel morphometric characteristics of the tumor growth pattern in lung adenocarcinoma can be potentially discovered from the reconstructed 3D histology image of a single FFPE lung specimen. To reconstruct the high-resolution 3D histology image of a whole tissue organ, the tissue organ is first sliced at a certain thickness. Each tissue slice is then cut into multiple tissue pieces and from each paraffin-embedded tissue piece thin tissue sections are subsequently cut. The initial step to achieve the $3 \mathrm{D}$ volume histology representation of the whole organ is therefore to reconstruct the 2D histology images of the tissue slices. Research groups $[22,23]$ presented a method to reconstruct the 2D histology image of whole prostate organ tissue slices. In their method, a whole prostate organ tissue slice was cut into $K=4$ tissue pieces, which were later embedded in paraffin blocks. Thin tissue sections were then cut from the top surfaces of the paraffin-embedded tissue blocks, i.e. 1 tissue section per tissue block, and then mounted on standard-size glass slides for hematoxylin and eosin (HE) staining. Using landmark points selected manually, the digital images of the 4 stained tissue sections were stitched together to create the $2 \mathrm{D}$ histology image of the prostate tissue slice.

The overall aim of our research study was to reconstruct the $3 \mathrm{D}$ volume histology image of the whole human brain tissue organ and to register the result to its postmortem ex vivo MR image. Since the surface area of a tissue 


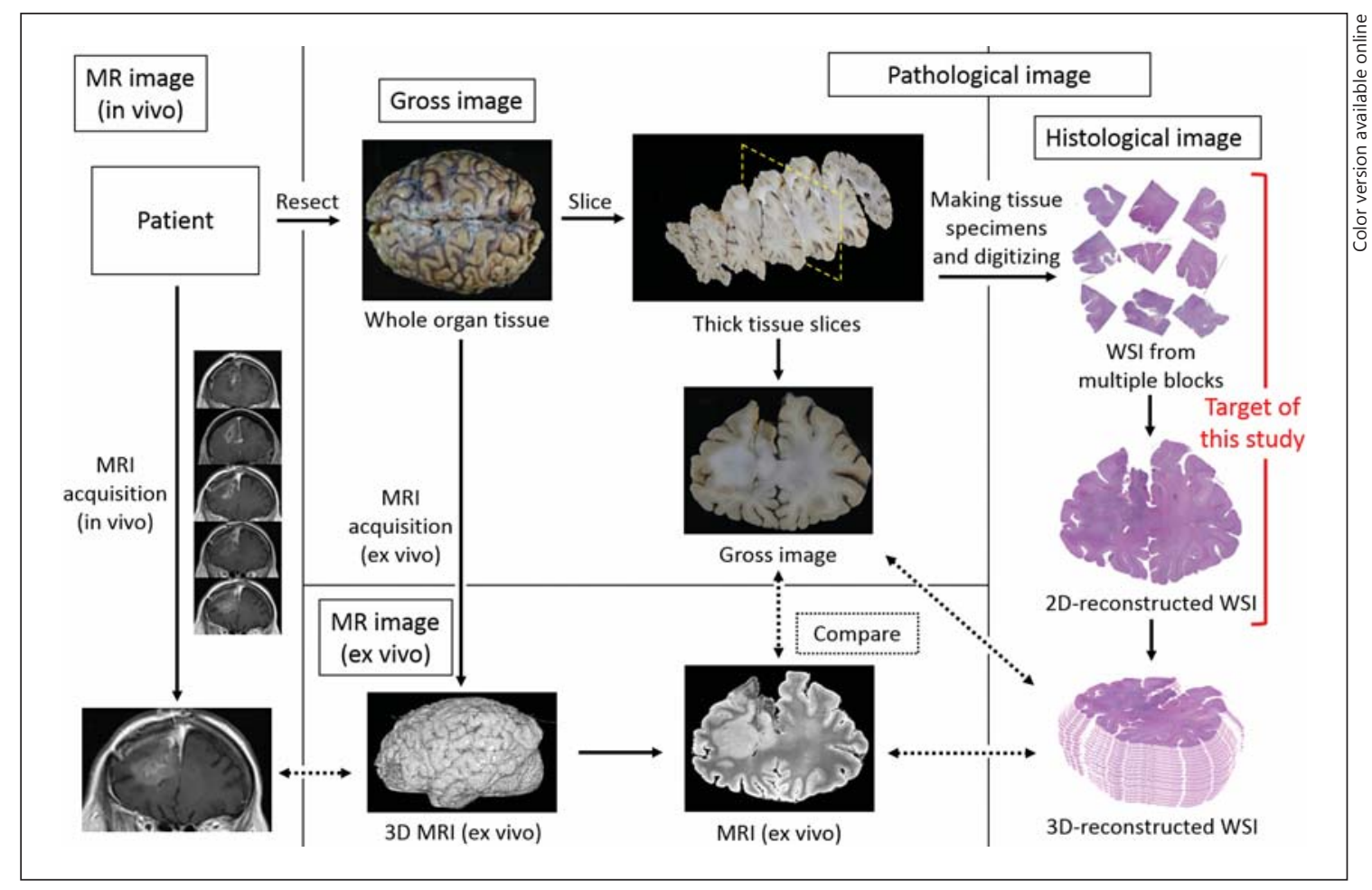

Fig. 1. Overall workflow of the 3D volume histology image reconstruction of the whole brain organ.

slice of the whole brain is larger compared to that of the prostate organ, a single organ's tissue slice could consist of multiple $(K>4)$ tissue pieces. Apparently, the technical level of difficulty in reconstructing the $2 \mathrm{D}$ histology image of a brain tissue slice is different from that of the prostate organ. Figure 1 shows various aspects of the overall workflow of our study. For this paper, we mainly focused on the $2 \mathrm{D}$ image reconstruction of a brain tissue slice and the development of a viewing system for observing the highresolution reconstructed 2D histology image efficiently with the standard PCs in our laboratories. To reconstruct the 2D histology image of a brain tissue slice, we proposed an image alignment algorithm that is tailored to the overall contour pattern of the brain's tissue slices.

\section{Materials and Methods}

Figure 2 outlines the processes involved to build the 2D highresolution histology image of a whole brain tissue slice. While processes 1-5 are accomplished in pathology laboratories (autopsy/ surgical and histological procedures), processes $6-8$ are performed at an imaging laboratory. In our work, we only used histology materials for which clinical diagnoses were already made. In the fol- lowing sections, we discuss the method to align the multiple histology images (8) to recreate the block face histology image of a whole brain tissue slice (3). We will also present the development of the $2 \mathrm{D}$ viewing system for observing the high-resolution reconstructed WSI of the whole tissue slice.

\section{Histology Processing}

The formalin-fixed specimen of the whole brain tissue organ was manually sliced in 5-mm-thick sections along the coronal plane; since the tissue slices were generated manually, we can expect some variation in their thickness. Each tissue slice was divided into 5- 15 tissue pieces, which were later embedded in paraffin wax to form tissue blocks. Tissue sections $5 \mu \mathrm{m}$ in thickness were sectioned from these tissue blocks and then mounted on standardsize glass slides for HE staining.

\section{Image Acquisition}

The gross images of the tissue slices were acquired using a digital camera and the high-resolution images $(0.46 \mu \mathrm{m} / \mathrm{pixel})$ of the histology slides of the $5-\mu \mathrm{m}$ HE-stained tissue sections with the Nanozoomer whole slide scanner. The Hamamatsu Nanozoomer API, NDPread was used to generate the thumbnail images of the high-resolution whole slide images. We note that the thumbnail images are representative images of the whole slide images at their lowest pixel resolution. Unless otherwise stated, we refer to the thumbnail version of a whole slide image of a tissue section as the histology image and the digital image of the whole brain's tissue slice as the gross image. 


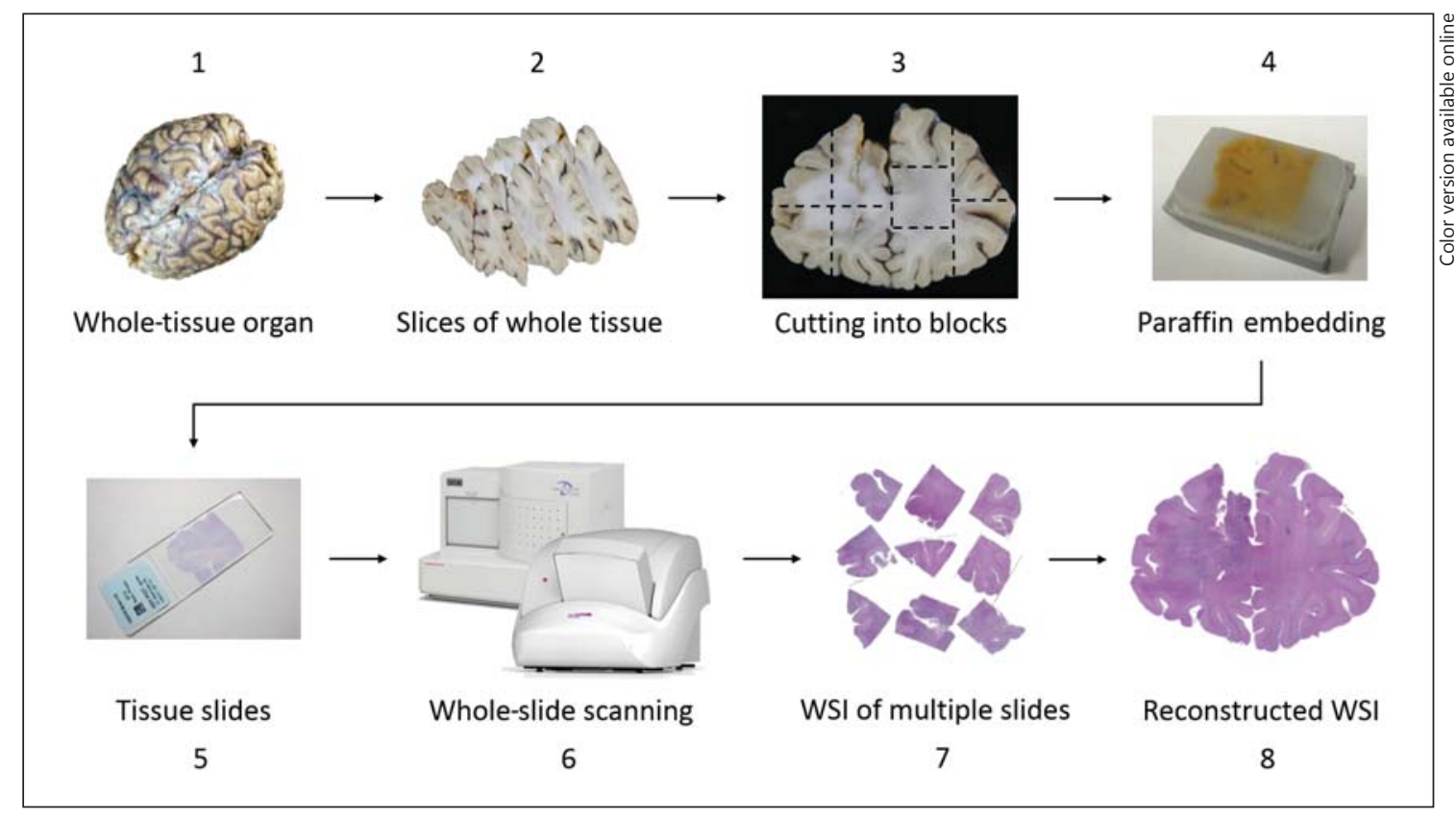

Fig. 2. Processes involved in reconstructing the high-resolution $2 \mathrm{D}$ histology image of a whole brain tissue slice.
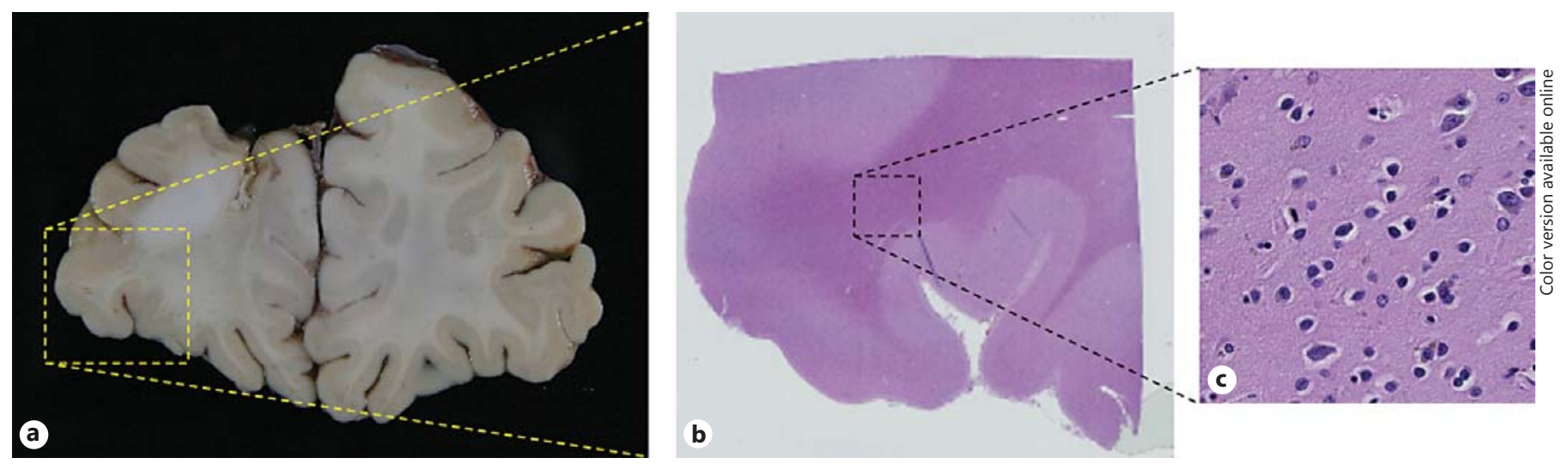

Fig. 3. Illustration on the effect of HE staining and the differences and similarities in the gray-level values of the unstained tissue slice image and stained histology images. a Gross image of a brain slice. b, c HE-stained histology images. b Thumbnail image of an HE-stained tissue section. c High-resolution image of an HE-stained tissue area.

\section{Image Color Preprocessing}

Chemical staining introduces color contrast between the different tissue structures. In HE-stained tissue, structures which have affinity to eosin will appear pink to red and those which have affinity to hematoxylin will appear blue to purple. The effect of HE staining is demonstrated in figure 3 . Figure 3 a displays the image of a $\sim 5$-mm-thick unstained tissue slice of human brain tissue. Figure $3 c$, on the other hand, displays the magnified image of a $\mathrm{HE}$-stained tissue slide of the region marked in figure $3 \mathrm{~b}$. Here, we can observe that the dark regions in the HE-stained image appear lighter in the gross image. To effectively perform image template matching between these two different sets of images, their original RGB colors were transformed to Hue saturation value (HSV) color format [24]. The correlation between the gray-level attributes of the gross- and HE-stained histology images is enhanced by representing the gross image by its value, $\mathrm{V}$, and the histology image by its saturation, S, color components in the HSV color space. Nonlinear contrast adjustment, in which the histogram of a given histology image is modified to match the histogram of its corresponding gross image using a lookup table (LUT), was further applied to the processed images. 


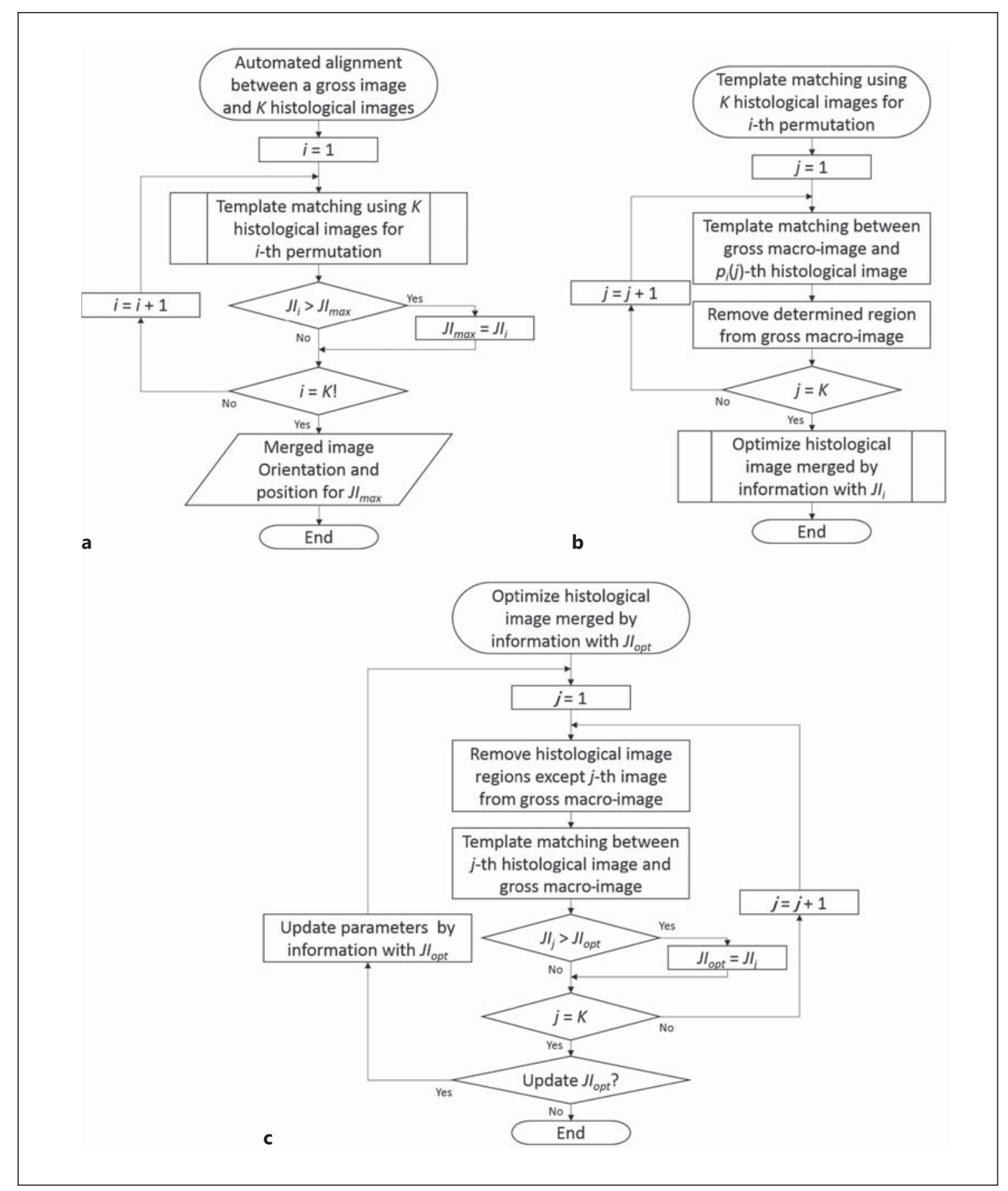

Fig. 4. Workflow of the proposed method to reconstruct the $2 \mathrm{D}$ histology image of a tissue slice composed of multiple histology images. a Flow of the entire algorithm. b Permutation-based template matching. c Iterative optimization process.

2D Histology Reconstruction of a Brain Tissue Slice

Figure $4 \mathrm{a}$ shows our proposed method to reconstruct the $2 \mathrm{D}$ histology image of a whole brain organ tissue slice. We note that the histology images, i.e. thumbnail images of the high-resolution whole slide images, have higher pixel resolution compared to the gross images of their tissue slices. Hence, the histology images were resampled to align their pixel resolution to the gross images of their tissue slices. The proposed method is composed of three major processes: (i) image template matching; (ii) optimization of the $2 \mathrm{D}$ histology image reconstruction result, and (iii) evaluation of the 
Table 1. Components of $p i(j)$ for the case with $K=3$, where pathological images are assigned to 1, 2 and 3 in advance

\begin{tabular}{llll}
\hline Sequence $i$ in $p_{\mathrm{i}}(j)$ & \multicolumn{4}{l}{ Processed image $j$ in $p_{\mathrm{i}}(j)$} \\
\cline { 2 - 4 } & 1 st & 2nd & 3rd \\
\hline 1 & No. 1 & No. 2 & No. 3 \\
2 & No. 1 & No. 3 & No. 2 \\
3 & No. 2 & No. 1 & No. 3 \\
5 & No. 2 & No. 3 & No. 1 \\
6 & No. 3 & No. 1 & No. 2 \\
& No. 3 & No. 2 & No. 1 \\
\hline
\end{tabular}

optimized $2 \mathrm{D}$ reconstructed image. For the image template matching, we designed the permutation-based approach described by the flowchart in figure $4 \mathrm{~b}$. The degree of similarity between the $2 \mathrm{D}$ reconstructed histology image and the gross image of its tissue slice is evaluated using the Jaccard index (JI). An iterative optimization procedure, illustrated in figure $4 \mathrm{c}$, is incorporated to optimize the image similarity between the gross image and the reconstructed $2 \mathrm{D}$ histology image.

\section{Permutation-Based Template Matching}

The $K$ histology images of the 5 - $\mu$ m-thick tissues, which were sectioned from $K$ multiple tissue pieces of a whole brain tissue slice, are arranged in $i=1,2, \ldots, K$ ! different orders generating $K$ ! different sets of sequenced images. The permutation-based template-matching algorithm can be described by considering a function $p_{i}(j)$ which outputs the $j$-th histology image of the $i$-th sequenced image set that has to be matched to the given gross image of its tissue slice. Template matching is repeatedly performed between the $j=1,2, \ldots, K$ histology images in all $i=1,2$, ..., $K$ ! sequenced image sets and its gross image counterpart. A version of the $2 \mathrm{D}$ histology image of the whole tissue slice section is derived by merging the matched $j=1,2, \ldots, K$ histology images in the $i$-th set. That is, the permutation-based template-matching procedure results to $K$ ! different $2 \mathrm{D}$ reconstructed histology images for every whole tissue slice section composed of $K$ multiple thin tissue sections. Table 1 illustrates the different image sequence sets ( $i$ ) and the possible orders of the images $(j)$ for the case when $K=3$.

In each template matching, the similarity between the gross and histology images is evaluated using normalized cross-correlation (NCC) expression:

$$
R_{N C C}=\frac{\sum_{n=0}^{N-1} \sum_{m=0}^{M-1} I(m, n) T(m, n)}{\sqrt{\sum_{n=0}^{N-1} \sum_{m=0}^{M-1} I(m, n)^{2} \times \sum_{n=0}^{N-1} \sum_{m=0}^{M-1} T(m, n)^{2}}} .
$$

In the expression above, $I(m, n)$ and $T(m, n)$ are the pixel intensities of the $M \times N$ source and reference (template) images at coordinates $(m, n)$, respectively. In this study, the source and the reference images correspond to the gross image of the tissue slice and the histology image, respectively. The image template matching is performed with histology images rotated at various angles at intervals of $1^{\circ}$. This interval can be changed coarsely, which would make computing time shorter. Then, the orientation and location of the $j$-th histology image with respect to the gross image at maximum correlation is noted, and the gray-level values of the image pixels within the region at which this occurred are set to zero. This region will be treated as part of the gross image background in the succeeding image template-matching procedure, for $\forall j=1,2, \ldots$, $K-1$ in $\forall i=1,2, \ldots, K !$.

\section{Image Alignment Optimization}

The permutation-based template matching discussed in the previous section is performed to derive several $2 \mathrm{D}$ reconstructed histology image versions of a given tissue slice. The template matching aligns $K$ histology images with respect to the gross image of its tissue slice. The estimated locations and orientations of the aligned histology images are further optimized to improve the match between the merged histology images and its gross tissue slice image. The similarity of each of these reconstructed 2D histology images to the gross image of its tissue slice is evaluated using the JI [25] (equation 2), which is defined as the ratio between the intersection and union of binary images $A$ and $B$, where $A$ is the source image and $B$ is the reference image. The JI will yield its maximum value, 1 , when the source image $A$ is the same as the reference image $B$, and 0 when the two binary images $A$ and $B$ are totally different. In our experiment, the binary images of the $2 \mathrm{D}$ reconstructed histology image $(A)$ and gross image $(B)$ were derived using discriminant analysis [26].

$$
J=\frac{|A \cap B|}{|A \cup B|} .
$$

Figure $4 \mathrm{c}$ shows the optimization procedure which applies template matching to $K$ histology images repeatedly in order. Each time the orientation of the $j$-th image of the $i$-th 2D reconstructed image is updated, a new JI is also calculated. The process continues iteratively until the difference between the current and previously calculated JI is 0 , i.e. there is no change in the JI.

\section{Development of a Viewer System for 2D High-Resolution \\ Images of Whole Tissue Sections}

The current whole slide image viewer is designed to only view the high-resolution image of a single tissue slide but not that of a whole section of a tissue slice, which has an image dimension 4-15 times to that of a single slide. We designed a viewer system to observe the high-resolution $2 \mathrm{D}$ reconstructed histology image of the whole brain tissue slice. Figure 5 shows the procedure of the viewing system for $2 \mathrm{D}$ whole tissue sections. The image viewer displays only the thumbnail image of the $2 \mathrm{D}$ reconstructed histology image of the whole tissue slice section and the original block-face gross image of the tissue slice. Since the $\mathrm{x}$-/y-coordinates of the displayed image are mapped to the high-resolution small image patches of the tissue slice, the user can click the mouse on any point on the displayed image to view the high-resolution image version of the specified area. In the implementation of the viewer system, the image patches are stored in our local server drive rather than in our local computer drives. 


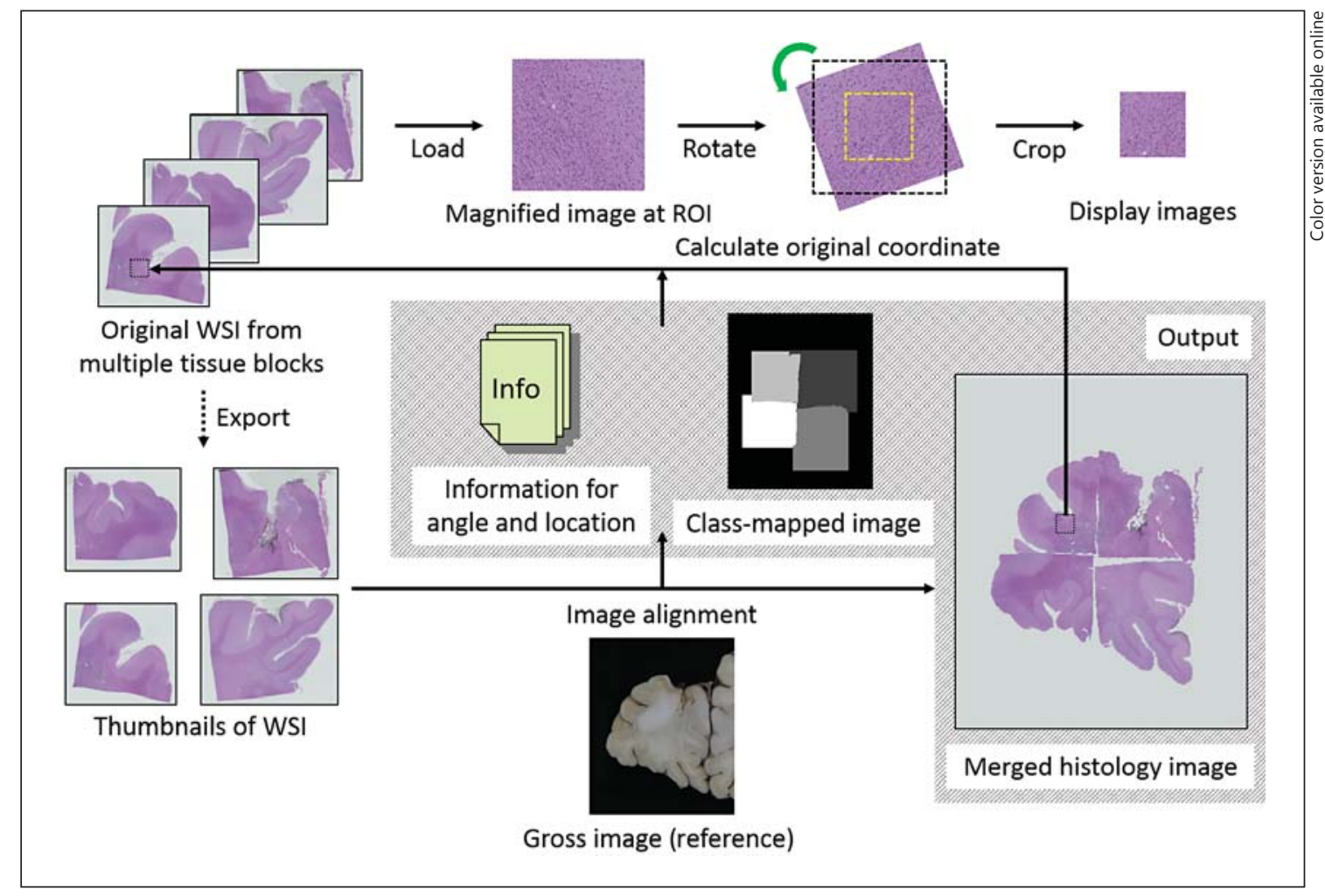

Fig. 5. Procedure of a viewing system for $2 \mathrm{D}$ high-resolution images of whole tissue slices. $\mathrm{ROI}=\mathrm{Regions}$ of interest.

\section{Experimental Results}

\section{Image Preprocessing}

The tissue specimen of the whole brain organ was sliced along the coronal plane. Brain tissue slices along this plane show demarcations between the right and left regions of the brain. An image processing algorithm was implemented to automatically identify the boundary between these regions from the gross images of the tissue slices prior to performing the image template matching. The algorithm considered the derivative of the gray-level intensities of the gross image across its width, i.e. along the $\mathrm{x}$-axis. The point at which the local minimum has the largest difference from the nearest local maximum was identified as the boundary between the left and right regions of the brain. Figure 6a shows the gross image of a brain coronal section, while the plot in figure $6 \mathrm{~b}$ illustrates the average gray-level profile of the image. In the plot, the y-axis corresponds to the average gray-level value of the image pixels measured at a point along the horizontal ( $\mathrm{x}$ ) axis.

The image color preprocessing detailed in the Section Image Color Preprocessing was applied to the HE-stained histology images. Figure 7 demonstrates the results of this procedure. It displays the original RGB color images of the HE-stained histology image (fig. 7a) and the gross image (fig. $7 d$ ). Figure $7 b$, e, on the other hand, displays the saturation (fig. $7 \mathrm{~b}$ ) and value component images (fig. 7e) of the RGB color images in figure $7 \mathrm{a}, \mathrm{d}$, and figure $7 \mathrm{c}$ illustrates the result of the contrast adjustment scheme we implemented to bring the gray-level histograms of the two sets of images even closer.

\section{D Histology Image Reconstruction of a Tissue Slice}

In this work, the histology slides were labeled as either belonging to the left or right regions of the brain tissue slice when they were handed to our laboratory. Hence, the permutation-based template matching described in $2 \mathrm{D}$ Histology Reconstruction of a Brain Tissue was independently performed on these regions. The reconstructed $2 \mathrm{D}$ histology image for the left and right brain regions are then merged to produce the final 2D reconstructed histology image of the whole tissue slice.

The similarity of the $2 \mathrm{D}$ reconstructed histology images of the whole brain's tissue slice produced by the per- 


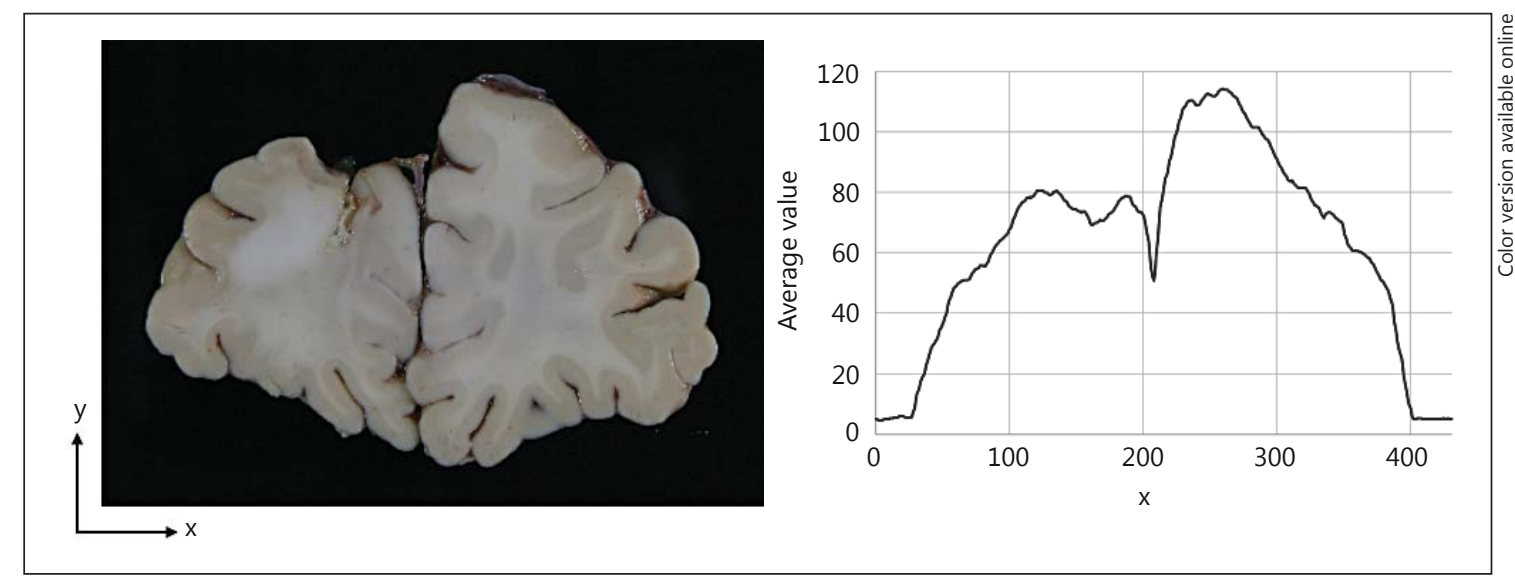

Fig. 6. Illustration of the average gray-level profile (b) of the gross image (a) of a tissue slice of a whole brain organ along the coronal plane.

Fig. 7. Image preprocessing of gross and histology images. a HE-stained histology image. $\mathbf{b}$ HSV saturation component of the HE-stained RGB color image. c Result of gray-level adjustment using LUT. d Gross image. e HSV value component of the gross image. $\mathbf{f}$ Magnified version of the value component image. The saturation and value of the images were determined by converting the original RGB color of the image into the HSV color space.
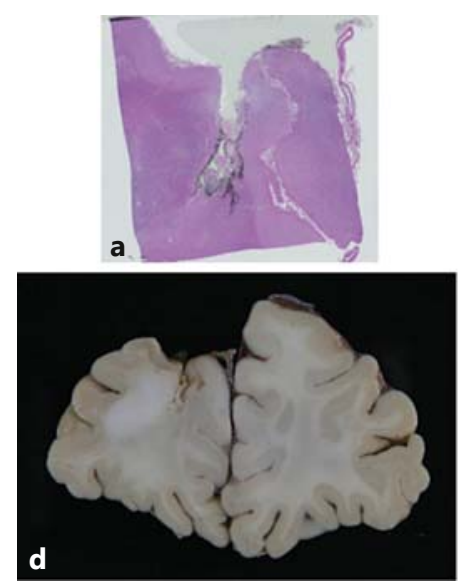
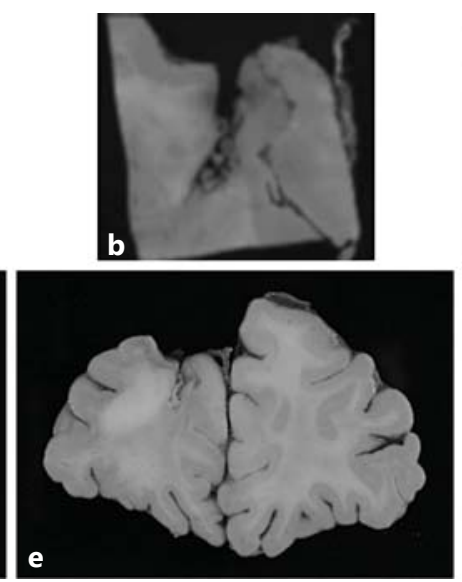
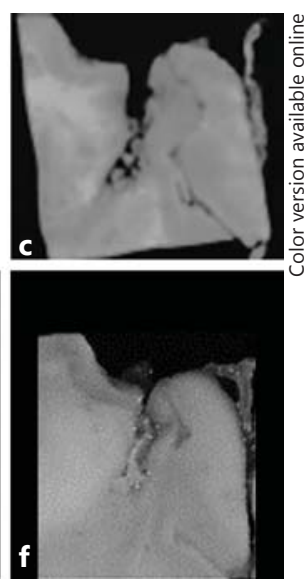

mutation-based template matching to the original gross image of the slices were optimized using the procedure detailed in the previously mentioned section wherein the JI (equation 2) was employed as the similarity criterion. The $2 \mathrm{D}$ reconstructed histology image which resulted in the highest JI was considered as the representative 2D histology image of the whole brain's tissue slice.

As a pilot study, we utilized 3 whole brain tissue slices which did not have deficient tissue blocks, although we had more datasets from other tissue slices. The assumption of this study is that a whole brain image can be reconstructed from its image fragments, and in the future tissue-making process should be also discussed for our objective. The numbers of tissue pieces included in each whole brain slice are shown in table 2 .

Figure 8 displays the results of this procedure to the images mentioned above. The gross images of the brain's
Table 2. Numbers of tissue blocks which are included in each whole brain tissue slice

\begin{tabular}{lllc}
\hline $\begin{array}{l}\text { Slice } \\
\text { No. }\end{array}$ & $\begin{array}{l}\text { Left } \\
\text { brain }\end{array}$ & $\begin{array}{l}\text { Right } \\
\text { brain }\end{array}$ & Total \\
\hline 1 & 3 & 3 & 6 \\
2 & 4 & 5 & 9 \\
3 & 5 & 5 & 10 \\
\hline
\end{tabular}

coronal sections are displayed in figure $8 \mathrm{a}$, while the stained histology images of the tissue sections cut from FFPE multiple tissue pieces of the tissue slice are displayed in figure $8 \mathrm{~b}$. Visually, the $2 \mathrm{D}$ reconstructed histology images (fig. 8c) share similar contours and shape patterns with the gross images (fig. 8a). The JI value of two 

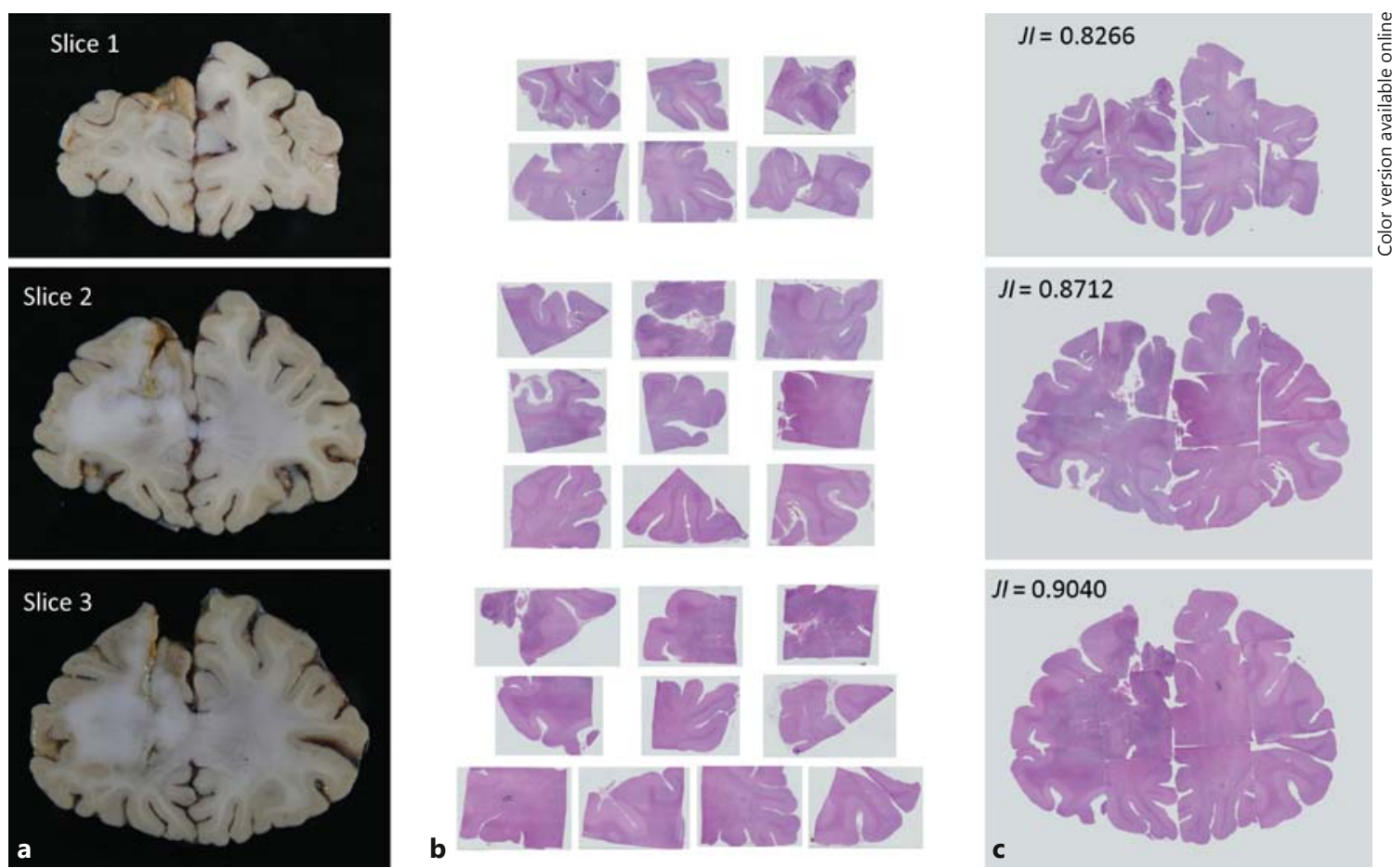

Fig. 8. The results of the proposed image alignment algorithm. a Gross images of whole brain tissue slices. b Histology images obtained from multiple tissue blocks. c The merged histology images with their JI.

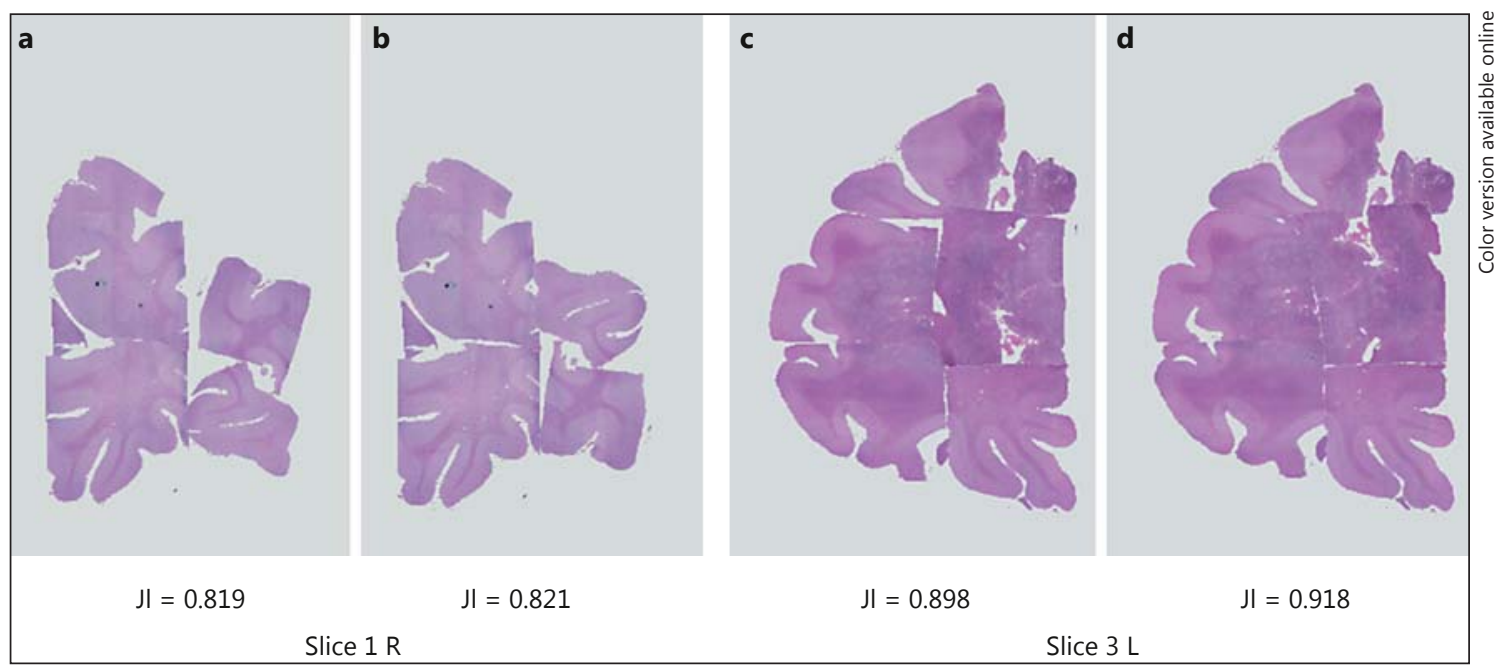

Fig. 9. Relationship between aligned results and JI to establish the adequacy of selecting a similarity index. Reconstructed images of worse alignment $(\mathbf{a}, \mathbf{c})$ and best alignment $(\mathbf{b}, \mathbf{d})$ for each slice, respectively.

perfectly matched images is 1 . Application of our image alignment method resulted in a minimum JI value of 0.83 and a maximum of 0.90 validating our visual assessment on the similarity between the 2D reconstructed histology image and brain tissue slice gross images.

Towards High-Resolution Whole-Organ 3D Histological Images

\section{Quantitative Evaluations}

The proposed algorithm employs NCC in template matching. In both histology and gross images, we can see the boundary and color difference between white matter and cortex, which is important information for compari- 


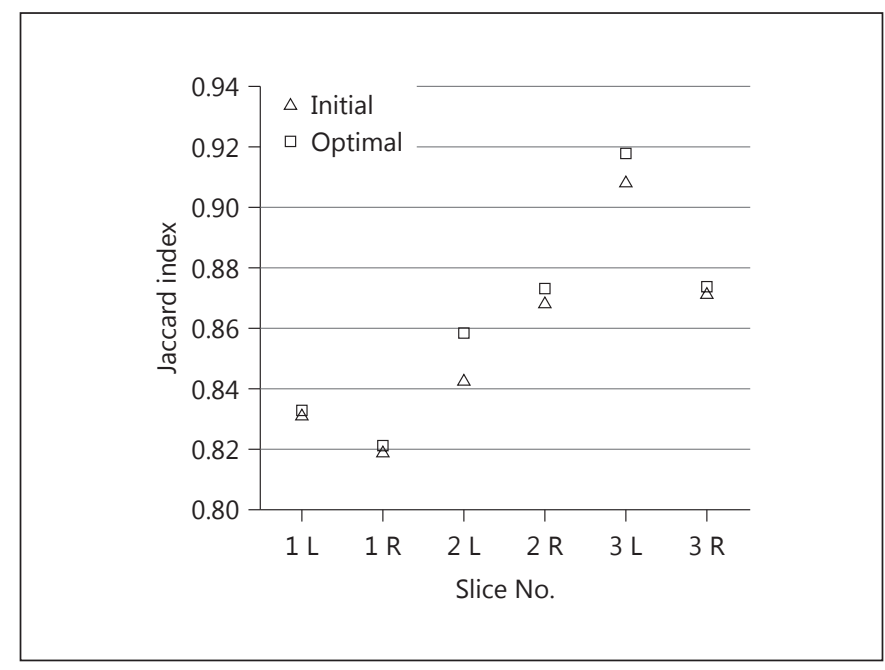

Fig. 10. Plots of maximum JI, which are calculated with and without optimization process. $\mathrm{x}$-axis and $\mathrm{y}$-axis indicate slice number and JI of the best alignment result, respectively.

son of similarity. Then, positions and orientations of each tissue block are roughly determined after initial alignment, and the processed region is quite restricted in the optimization step. For fine-tuning, we selected JI to compare the degree of coincidence. Figure 9 shows the relationship between aligned results and the JI to establish the adequacy of selecting a similarity index. We can see that the merged images with lower JI have some misalignment in the results. Furthermore, plots of maximum JIs, which are calculated with and without optimization process, are shown in figure 10. The plots indicate the effectiveness of the optimization process from the relationship between increment of similarity indices and figure 9. As mentioned above, we have confirmed the validity of the similarity index and optimization process.

\section{Viewer of the 2D Histology Image of Brain Tissue Slices}

A snap shot of the $2 \mathrm{D}$ whole slice image viewer, which we made to view the high-resolution $2 \mathrm{D}$ reconstructed histology image of a brain tissue slice by Visual Basic, a programming language, is shown in figure 11 . This viewer does not require a whole tissue slice as one image. The higher-resolution image of $768 \times 768$ pixel of a point selected by the user from either the gross or low-resolution histology image through clicking or/and rolling the mouse is displayed in a separate window like a common WSI viewer. Since all WSIs belonging to a slice stay separately in the original place and the viewer loads only the appropriate part of the corresponding WSI(s) with the requested resolution and location using the output information which described 2D Histology Image Reconstruction of a Tissue Slice, the viewer does not require any special computer or memory to view a whole slice.

\section{Discussion}

Histology image analysis remains the gold standard for tissue characterization [27]. Findings in conventional MRI should be validated with histopathology imaging [28]. The potential of 3D histology imaging for closer investigation of changes in the morphology of a tissue structure in relation to a specific disease has been highlighted in a number of papers [29-30]. The authors discussed the potential of the $3 \mathrm{D}$ volume representation of a tissue organ to define more accurate histological and cytological parameters for further understanding of the disease. The computational difficulty in building the 3D volume histology image of a tissue organ increases as the $3 \mathrm{D}$ volumetric size of the organ increases. We note that in order for us to observe the needed cellular details of the tissue organ from its $3 \mathrm{D}$ reconstructed histology, it is ideal that we utilize thin tissue sections. This will require to divide the whole tissue slice of the organ into $K$ multiple tissue pieces. The initial step then to build the 3D histology image volume of the whole tissue organ is to piece the thinly sectioned tissues together to reconstruct the high-resolution 2D histology image of sectioned tissue slices. In this paper, we have introduced a permutation-based image template-matching method to reconstruct the $2 \mathrm{D}$ histology image of the whole brain's tissue slice. An optimization procedure that utilized the JI was further incorporated to improve the similarity between the reconstructed $2 \mathrm{D}$ histology image and the gross image of its tissue slice. Results of our initial experiments showed that this approach can satisfactorily reconstruct the $2 \mathrm{D}$ histology image of the whole brain's tissue slice.

We have also presented an initial implementation of the viewer to view the reconstructed high-resolution $2 \mathrm{D}$ histology image of a brain tissue slice. The low resolution, the $2 \mathrm{D}$ reconstructed histology or the gross image of the tissue slice is the navigator to display a higher resolution of an image of an area. It is the same concept with a general WSI viewer; it works as a whole slice viewer.

\section{Comparison with Other Methods}

A method to reconstruct the 2D whole-mount histology image of a prostate tissue was introduced previously 
Fig. 11. Snap image of the initial design of the whole slice image viewer.

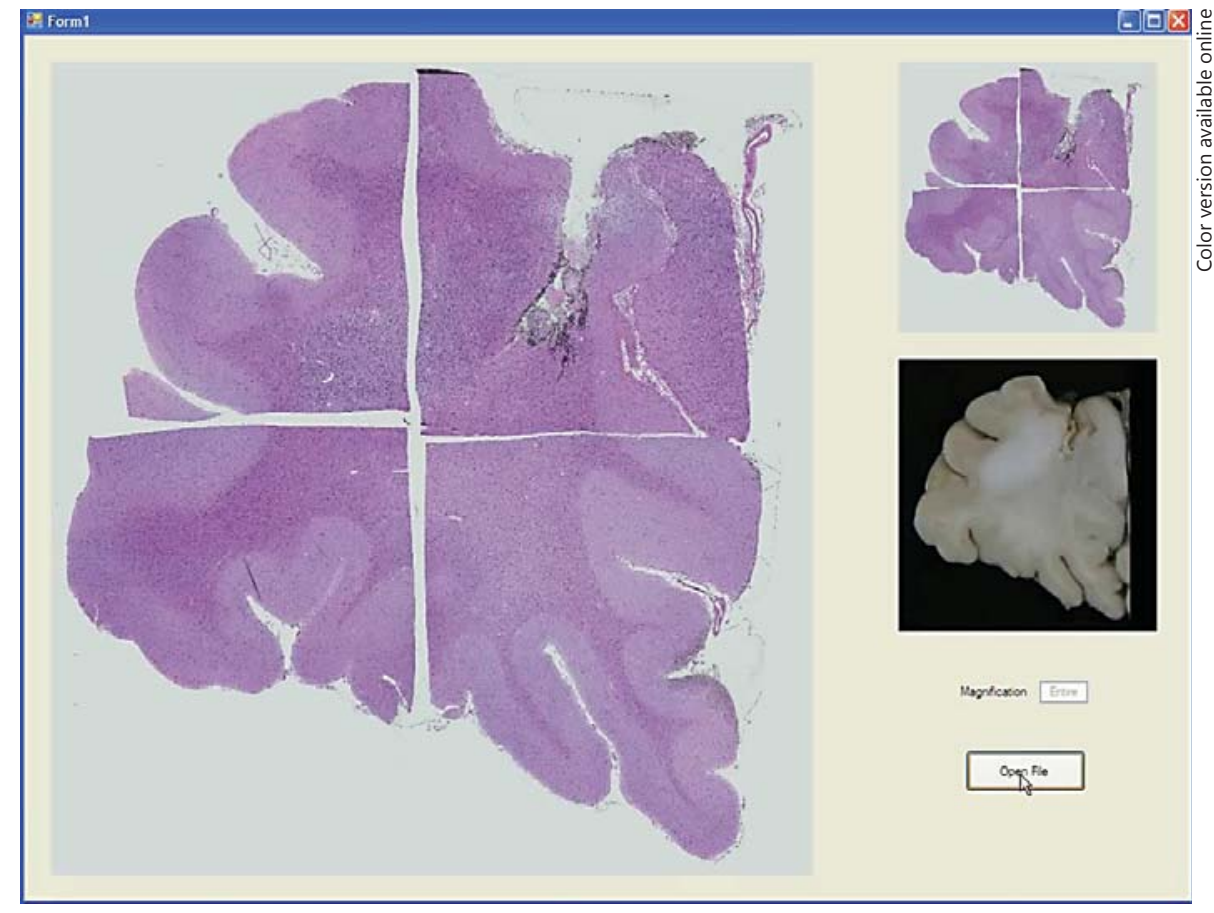

[23]. In this method, the whole prostate tissue was divided into 4 quadrants, and then whole-slide images of the quadrant tissues were reassembled to produce a pseudo whole-mount histology section image. The $2 \mathrm{D}$ reconstruction workflow involves 3 general steps: (i) loading the images; (ii) manual selection of fiducials from the edges of 2 quadrant images, and, with the given fiducials, (iii) stitching is done using affine transformation. The stitching procedure is repeated until all the quadrants are aligned. This workflow indeed incorporates inputs from clinicians to ultimately produce the pseudo whole-mount histology section. While the work presented herein shares a similar objective with the work presented by Toth et al. [23], the approaches that were adopted to reconstruct the $2 \mathrm{D}$ whole-mount tissue image differ. The present method does not require clinicians to select fiducials by which to base the alignment of the histology images - histology images are automatically aligned and optimized. The procedure of our 2D whole-mount histology image reconstruction is illustrated in figure 5 , and optimization of the computer memory usage is also an integratal part of the design. Since this was our initial work on the $2 \mathrm{D}$ reconstruction of whole mount tissue, the software implementation was limited for desktop or laptop computers.

The ultimate goal of our present work was to reconstruct the high-resolution 3D histology image volume of the whole human brain. This same goal was shared by the work by Amunts et al. [20]. In their work, a large-scale microtome was used to cut the complete paraffin-embedded human brain at $20-\mu \mathrm{m}$ thickness. The sectioned tissues were then mounted on large glass slides, stained and digitized using a flatbed scanner. In contrast, in our method, the whole slice of brain tissue sections $\sim 5 \mathrm{~mm}$ in thickness was cut into smaller tissue pieces. These tissue pieces were later embedded in paraffin, sectioned at 5- $\mu \mathrm{m}$ thickness and digitized using a high-resolution whole-slide scanner. Thus, our approach to the 3D histology reconstruction of the whole human brain tissue organ adapts to the current histology workflow we adopted, and since it uses thinner tissue sections, i.e. $5 \mu \mathrm{m}$, as opposed to the 20 $\mu \mathrm{m}$ used previously [20], the resultant 3D histology image volume shows more cellular details. The principal reason for the use of the rigid image registration is not to deform such cellular details in higher magnification images.

\section{Limitations and Future Work}

The $2 \mathrm{D}$ reconstructed histology image of the brain's tissue slice exhibits gaps between tissue section images. These gaps could be ascribed to the limitations of our method, imperfections in tissue processing and deformations of the tissue itself during tissue processing and cutting [27]. We note that the tissue pieces, from which the stained tissue sections were cut, were manually cut from whole brain's tissue slices. It is possible that small parts of 
the tissue pieces were lost during this process. Also, some of the tissue sections were not ideally mounted onto glass slides. Some parts of the sectioned tissues fell off from the edges of the glass slides and could not be imaged by the whole slide scanner. To achieve the ideal $2 \mathrm{D}$ histology image of the whole section of a brain's tissue slice, it is important that the stated issues are addressed.

The 2D reconstruction method of the histology images of the brain's tissue slice that we proposed in this paper is not yet fully automated. In this work, the stained tissue slides were initially labeled as either belonging to the left or right part of the brain. The $2 \mathrm{D}$ reconstruction algorithm that we designed works on the assumption that the histology slides that will be handed to us are labeled as either belonging to the right or left regions of the brain section, which is not necessarily true in practical scenario. Part of our next work is to design a 2D histology image reconstruction algorithm that is void of this assumption.

The whole slice image viewer for the $2 \mathrm{D}$ reconstructed image of a brain slice realizes our idea on how we could browse the high-resolution 2D histology images of a brain slice with effective use of the power of a standard computer.

\section{Conclusion}

The 3D histology image reconstruction of the whole brain organ will have a significant impact on new discoveries in medicine. It will help us to understand the histology features of the brain and particularly their correlation to brain diseases. To render the 3D histology volume of a whole brain organ, we need to (i) reconstruct the $2 \mathrm{D}$ histology image of the brain slices and (ii) align the series of 2D reconstructed images of the brain slices. We proposed in this paper a method to address (i). Our experimental results showed that with the method presented we can reconstruct the 2D histology image of brain slices fairly well. The results also revealed the important role of tissue processing for accurate reconstruction of the $3 \mathrm{D}$ histology of the brain.

\section{References}

1 Maes F, Collignon A, Vandermeulen D, Marchal G, Suetens P: Multimodality image registration by maximization of mutual information. IEEE Trans Med Imaging 1997;16: 187-198.

-2 Zana F, Klein JC: A multimodal registration algorithm of eye fundus images using vessels detection and Hough transform. IEEE Trans Med Imaging 1999;18:419-428.

-3 Can A, Bello M, Cline HE, Tao X, Ginty F, Sood A, Gerdes M, Montalto M: Multi-modal imaging of histological tissue sections. Proc 5th IEEE International Symposium on Biomedical imaging: From Nano to Macro, Paris, 2008, pp 288-291.

4 Weinstein RS, Descour MR, Liang C, Barker G, Scott KM, Richter L, Krupinski EA, Bhattacharyya AK, Davis JR, Graham AR, Rennels M, Russum WC, Goodall JF, Zhou P, Olszak AG, Williams BH, Wyant JC, Bartels PH: An array microscope for ultrarapid virtual slide processing and telepathology. Design, fabrication, and validation study. Hum Pathol 2004;35:1303-1314.

5 Ho J, Parwani AV, Jukic DM, Yagi Y, Anthony L, Gilbertson JR: Use of whole slide imaging in surgical pathology quality assurance: design and pilot validation studies. Hum Pathol 2006;37:322-331.

6 Gilbertson JR, Ho J, Anthony L, Jukic DM, Yagi Y, Parwani AV: Primary histologic diagnosis using automated whole slide imaging: a validation study. BMC Clin Pathol 2006;6:4.
7 Rojo MG, García GB, Mateos CP, García JG, Vicente MC: Critical comparison of $31 \mathrm{com}$ mercially available digital slide systems in pathology. Int J Surg Pathol 2006;14:285-305.

8 Weinstein RS, Graham AR, Richter LC, Barker GP, Krupinski EA, Lopez AM, Erps KA, Bhattacharyya AK, Yagi Y, Gilbertson JR: Overview of telepathology, virtual microscopy, and whole slide imaging: prospects for the future. Hum Pathol 2009;40:1057-1069.

$\checkmark 9$ Wilbur DC, Madi K, Colvin RB, Duncan LM, Faquin WC, Ferry JA, Frosch MP, Houser SL, Kradin RL, Lauwers GY, Louis DN, Mark EJ, Mino-Kenudson M, Misdraji J, Nielsen GP, Pitman MB, Rosenberg AE, Smith RN, Sohani AR, Stone JR, Tambouret RH, Wu C-L, Young RH, Zembowicz A, Klietmann W: Whole-slide imaging digital pathology as a platform for teleconsultation: a pilot study using paired subspecialist correlations. Arch Pathol Lab Med 2009;133:1949-1953.

10 Sertel O, Kong J, Shimada H, Catalyurek UV, Saltz JH, Gurcan MN: Computer-aided prognosis of neuroblastoma on whole-slide images: classification of stromal development. Pattern Recognit 2009;42:1093-1103.

11 Tuominen VJ, Ruotoistenmäki S, Viitanen A, Jumppanen M, Isola J: ImmunoRatio: a publicly available web application for quantitative image analysis of estrogen receptor (ER), progesterone receptor (PR), and Ki-67. Breast Cancer Res 2010;12:R56.
12 Huang C-H, Veillard A, Roux L, Loménie N, Racoceanu D: Time-efficient sparse analysis of histopathological whole slide images. Comput Med Imaging Graph 2011;35:579-591.

13 Onozato ML, Klepeis VE, Yagi Y, Mino-Kenudson M: A role of three-dimensional (3D)reconstruction in the classification of lung adenocarcinoma. Anal Cell Pathol (Amst) 2012; 35:79-84.

14 Gibb M, Burton RAB, Bollensdorff C, Afonso C, Mansoori T, Schotten U, Gavaghan DJ, Rodriguez B, Schneider JE, Kohl P, Grau V: Resolving the three-dimensional histology of the heart; in Gilbert D, Heiner M (eds): Computational Methods in Systems Biology. Berlin, Springer, 2012, pp 2-16.

15 Onozato ML, Kovach AE, Yeap BY, MoralesOyarvide V, Klepeis VE, Tammireddy S, Heist RS, Mark EJ, Dias-Santagata D, Iafrate AJ, Yagi Y, Mino-Kenudson M: Tumor islands in resected early-stage lung adenocarcinomas are associated with unique clinicopathologic and molecular characteristics and worse prognosis. Am J Surg Pathol 2013;37: 287-294.

16 Song Y, Treanor D, Bulpitt AJ, Magee DR: 3D reconstruction of multiple stained histology images. J Pathol Inform 2013;4(suppl):S7.

17 Norton K-A, Namazi S, Barnard N, Fujibayashi M, Bhanot G, Ganesan G, Iyatomi $\mathrm{H}$, Ogawa K, Shinbrot T: Automated reconstruction algorithm for identification of $3 \mathrm{D}$ architectures of cribriform ductal carcinoma in situ. PLoS One 2012;7:e44011. 
18 Onozato ML, Hammond S, Merren M, Yagi Y: Evaluation of a completely automated tissue-sectioning machine for paraffin blocks. J Clin Pathol 2013;66:151-154.

$\checkmark 19$ Fujisawa S, Yarilin D, Fan N, Turkekul M, Xu $\mathrm{K}$, Barlas A, Manova-Todorova K: Understanding the three-dimensional world from two-dimensional immunofluorescent adjacent sections. J Pathol Inform 2015;6:27.

20 Amunts K, Lepage C, Borgeat L, Mohlberg H, Dickscheid T, Rousseau M-É, Bludau S, Bazin P-L, Lewis LB, Oros-Peusquens A-M, Shah NJ, Lippert T, Zilles K, Evans AC: BigBrain: an ultrahigh-resolution 3D human brain model. Science 2013;340:1472-1475.

-21 Yagi Y, Gilbertson JR: A relationship between slide quality and image quality in whole slide imaging (WSI). Diagn Pathol 2008;3(suppl 1):S12.

22 Chappelow J, Tomaszewski JE, Feldman M, Shih N, Madabhushi A: HistoStitcher ${ }^{(\mathbb{(})}$ : an interactive program for accurate and rapid reconstruction of digitized whole histological sections from tissue fragments. Comput Med Imaging Graph 2011;35:557-567.

23 Toth RJ, Shih N, Tomaszewski JE, Feldman MD, Kutter O, Yu DN, Paulus JC, Paladini G, Madabhushi A: Histostitcher ${ }^{\mathrm{TM}}$ : an informatics software platform for reconstructing whole-mount prostate histology using the extensible imaging platform framework. J Pathol Inform 2014;5:8.

24 Tsamoura E, Pitas I: Automatic color based reassembly of fragmented images and paintings. IEEE Trans Image Process 2010;19:680-690.

25 Amiri S, Movahedi MM, Kazemi K, Parsaei H: An automated MR image segmentation system using multi-layer perceptron neural network. J Biomed Phys Eng 2013;3:115-122.

26 Citak-Er F, Vural M, Acar O, Esen T, Onay A, Ozturk-Isik E: Final Gleason score prediction using discriminant analysis and support vec- tor machine based on preoperative multiparametric MR imaging of prostate cancer at 3T. Biomed Res Int 2014;2014:690787.

27 Caplan J, Niethammer M, Taylor RM, Czymmek KJ: The power of correlative microscopy: multi-modal, multi-scale, multi-dimensional. Curr Opin Struct Biol 2011;21:686-693.

28 Beare R, Richards K, Murphy S, Petrou S, Reutens D: An assessment of methods for aligning two-dimensional microscope sections to create image volumes. J Neurosci Methods 2008;170:332-344.

29 Eriksson SH, Free SL, Thom M, Harkness W, Sisodiya SM, Duncan JS: Reliable registration of preoperative MRI with histopathology after temporal lobe resections. Epilepsia 2005; 46:1646-1653.

30 Ourselin S, Roche A, Subsol G, Pennec X, Ayache N: Reconstructing a 3D structure from serial histological sections. Image Vision Computing 2001;19:25-31. 